\title{
Lesión hepática inducida por fármacos (DILI) de tipo colestásico secundaria al uso de levodopa/carbidopa
}

\section{Cholestatic drug-induced liver injury (DILI) secondary to the use of levodopa/carbidopa}

\author{
Juan D. Díaz-García ${ }^{1 *}$, Jazmín T. Pozos-López ${ }^{1,2}$, Areli Cruz-Trujillo ${ }^{2}$, Cindy A. Leal-Ahumada ${ }^{1}$, \\ Abel Fuentes-Venegas ${ }^{1}$ y Jacqueline Córdova-Gallardo ${ }^{4}$
}

${ }^{1}$ Servicio de Medicina Interna; ${ }^{2}$ Servicio de Geriatría; ${ }^{3}$ Servicio de Farmacovigilancia; ${ }^{4}$ Servicio de Hepatología. Hospital General Dr. Manuel Gea González, Ciudad de México, México

\begin{abstract}
Drug-induced liver injury (DILI) is a rare condition. There are three patterns of damage: hepatocellular, cholestatic and mixed, with the cholestatic pattern representing $20-40 \%$ of cases. Its manifestations range from biochemical alterations in the absence of symptoms, to acute liver failure and chronic liver damage. The diagnosis is reached by exclusion based on circumstantial evidence. In most cases, the patient's condition improves with the withdrawal of the medication responsible for the damage. A case of hepatic toxicity induced by levodopa/ carbidopa in an 84-year-old male patient is presented, with its respective approach followed and progress.
\end{abstract}

Key words: DILI. Acute liver failure. Cholestasis. Levodopa/carbidopa.

\section{RESUMEN}

La enfermedad hepática inducida por fármacos (DILI, drug induced liver injury) es un trastorno poco frecuente. Existen tres patrones de daño: hepatocelular, colestásico y mixto, representando el patrón colestásico el 20-40\% de los casos. Sus manifestaciones van desde las alteraciones bioquímicas en ausencia de síntomas hasta la insuficiencia hepática aguda y el daño hepático crónico. El diagnóstico es de exclusión, basado en evidencia circunstancial. En la mayoría de los casos el cuadro del paciente mejora con la retirada del medicamento responsable del daño. Se presenta un caso de toxicidad hepática inducida por levodopa/carbidopa en un paciente de sexo masculino de 84 años de edad, con su respectivo abordaje y evolución.

Palabras clave: DILI. Insuficiencia hepática aguda. Colestasis. Levodopa/carbidopa.

\section{Correspondence:}

*Juan D. Díaz-García

E-mail: judan_digar@hotmail.com
Date of reception: 01-08-2019

Date of acceptance: 12-06-2020

DOI: 10.24875/HMCM.19000222
Available online: $18-11-2020$

Hosp Med Clin Manag. 2020;13:124-9

2604-0018 / ( 2020 Mexican Regional Hospitals of High Specialty and Federal Hospitals. Published by Permanyer. This is an open access article under the CC BY-NC-ND license (http://creativecommons.org/licenses/by-nc-nd/4.0/). 


\section{INTRODUCCIÓN}

La enfermedad hepática inducida por medicamentos (DILI, drug induced liver injury) es una causa poco frecuente de este tipo de enfermedad en la población general, dando cuenta de menos del $1 \%$ de los pacientes hospitalizados con ictericia ${ }^{1,2}$. Sin embargo, esta entidad explica entre el 40 y el $50 \%$ de las causas de insuficiencia hepática aguda ${ }^{3,4}$, y se estima que a los seis meses del inicio del cuadro clínico uno de cada 10 pacientes muere o requiere trasplante hepático, y uno de cada cinco desarrolla enfermedad hepática crónica ${ }^{5}$.

Se han definido tres patrones de lesión de DILI: colestásico, hepatocelular y mixto. El primero se caracteriza por una elevación de la fosfatasa alcalina (FA) dos veces mayor del límite superior normal y/o una $R$ menor o igual de 2, siendo $\mathrm{R}$ la relación entre la alanino aminotransferasa (ALT) y la FA (Fig. 1). El patrón hepatocelular se define como ALT dos veces mayor del límite superior normal y/o R > 5. El patrón mixto es ALT dos veces mayor del límite superior normal, con $R$ entre 2 y $5^{6-10}$.

El 20-40\% de los casos de DILI se presentan con patrón colestásico, en comparación con un 40-78\% para el hepatocelular y un $12-20 \%$ para el mixto ${ }^{9}$. La presentación colestásica, a pesar de tener una mejor tasa de supervivencia en general, tiene también una tasa menor de progresión favorable del perfil bioquímico hepático y un mayor riesgo de desarrollo de enfermedad hepática crónica ${ }^{10}$.

\section{CASO CLÍNICO}

Hombre de 84 años de edad con antecedentes de hipertensión arterial sistémica en tratamiento con losartán $50 \mathrm{mg}$ vía oral cada 12 horas y amlodipino $5 \mathrm{mg}$ vía oral cada 24 horas desde el año 1990, y enfermedad de Parkinson en tratamiento con levodopa (I-dopa)/carbidopa $250 \mathrm{mg} / 25 \mathrm{mg}$ vía oral cada 12 horas desde el día 20 febrero de 2019. Ingresó por cuadro clínico de 12 días de evolución consistente en dolor abdominal difuso de intensidad en la escala visual analógica 7/10, no irradiado, asociado a distensión generalizada, el cual se exacerbó con la ingesta de alimentos.

A su ingreso a la unidad de urgencias el día 22 de marzo de 2019 presentó signos vitales dentro de los parámetros normales. Se solicitaron exámenes de

\section{ALT (paciente) / ALT (límite superior normal) \\ $\mathbf{R}=$ \\ R= FA (paciente) / FA (límite superior normal)}

Figura 1. Valor $\mathrm{R}$ para patrones de lesión hepática inducida por fármacos. ALT: alanino aminotransferasa; FA: fosfatasa alcalina.

laboratorio, que se encontraron sin alteraciones y con las siguientes pruebas de función hepática: bilirrubina total $0.50 \mathrm{mg} / \mathrm{dl}(0.1-1.0)$, bilirrubina directa $0.22 \mathrm{mg} / \mathrm{dl}$ (0.1-0.25), ALT $20 \mathrm{UI} / \mathrm{I}(10-40)$, aspartato aminotransferasa (AST) $15 \mathrm{UI} / \mathrm{I}$ (15-41), gamma-glutamil transferasa (GGT) 20 UI/I (9-40) y FA 50 UI/I (38-126). Fue valorado por el servicio de cirugía general, el cual solicitó una tomografía computarizada de abdomen contrastada que reportó datos de enfermedad diverticular, iniciándose manejo conservador con ayuno, líquidos parenterales y colocación de sonda nasogástrica, con resolución en las primeras 48 horas. Dado que presentó temblor en reposo e inestabilidad postural, se interconsultó al servicio de neurología el día 24 de marzo, que incrementó la dosis de I-dopa/carbidopa a $250 \mathrm{mg} / 25 \mathrm{mg}$ vía oral cada 8 horas con posterior mejoría de la sintomatología, egresando el día 28 de marzo de 2019. Sin embargo, el día 30 de marzo presentó cuadro clínico caracterizado por ictericia mucotegumentaria, prurito generalizado y náuseas, acudiendo nuevamente a nuestro hospital. Dentro de los paraclínicos destacaron los siguientes: bilirrubina total $2.80 \mathrm{mg} / \mathrm{dl}(0.1-1.0)$, bilirrubina directa $1.28 \mathrm{mg} / \mathrm{dl}$ (0.1-0.25), ALT $11 \mathrm{UI} / \mathrm{I}$ (10-40), AST $125 \mathrm{UI} / \mathrm{l}$ (15-41), GGT 1,280 UI/I (9-40) y FA 1843 UI/I (38-126). Se realizó ultrasonografía de hígado y vías biliares sin evidenciar dilatación de la vía biliar, con morfología hepática normal y colangiorresonancia magnética sin alteraciones. Se solicitaron pruebas analíticas especiales como parte del abordaje de una colestasis, donde se reportaron: serología de virus hepatotropos (VHA, VHB, VHC y VHE) negativas, citomegalovirus y virus de Epstein-Barr negativos. Perfil serológico de hepatopatías autoinmunes con reporte de anticuerpos antinucleares mediante inmunofluorescencia indirecta, anticuerpos antimitocondriales, anticuerpos antimúsculo liso y anti-LKM1 negativos. Ante la sospecha de un DILI secundario al incremento de la dosis de I-dopa/carbidopa, se calculó el valor $\mathrm{R}$ en 0.21 , lo que indica un patrón de lesión colestásico. Se calculó la puntuación de la escala de CIOMS/RUCAM (Roussel Uclaf Causality Assesment Method, o Método de Evaluación de Causalidad Roussel Uclaf) de 9 puntos, indicando un DILI definitivo. Por ello se suspendió 
Tabla 1. Pruebas de función hepática: descenso gradual de GGT y FA posterior a la suspensión de levodopa/carbidopa (5 de abril de 2019)

\begin{tabular}{|c|c|c|c|c|c|c|c|}
\hline Fecha/parámetro & GGT (UI/I) & FA (UI/I) & $\begin{array}{l}\text { Bilirrubina total } \\
\text { (mg/dl) }\end{array}$ & $\begin{array}{c}\text { Bilirrubina } \\
\text { directa }(\mathrm{mg} / \mathrm{dl})\end{array}$ & $\begin{array}{c}\text { Bilirrubina } \\
\text { indirecta }(\mathrm{mg} / \mathrm{dl})\end{array}$ & ALT (UI/I) & AST (UI/I) \\
\hline 22/marzo/2019 & 20 & 50 & 0.50 & 0.22 & 0.28 & 20 & 15 \\
\hline 30/marzo/2019 & 1,280 & 1,243 & 2.80 & 1.28 & 1.52 & 11 & 125 \\
\hline 2/abril/2019 & 1,494 & 1,440 & 2.84 & 1.30 & 1.54 & 20 & 122 \\
\hline 4/abril/2019 & 1,693 & 2,002 & 1.73 & 0.81 & 0.92 & 9 & 136 \\
\hline 5/abril/2019 & 1,708 & 2,148 & 1.48 & 0.66 & 0.82 & 23 & 126 \\
\hline 7/abril/2019 & 1,381 & 1,772 & 1.25 & 0.40 & 0.85 & 41 & 86 \\
\hline 9/abril/2019 & 1,280 & 1,425 & 1.37 & 0.72 & 0.65 & 31 & 82 \\
\hline 11/abril/2019 & 949 & 1,061 & 0.93 & 0.44 & 0.49 & 24 & 60 \\
\hline 15/abril/2019 & 826 & 902 & 1.2 & 0.63 & 0.61 & 40 & 111 \\
\hline 18/abril/2019 & 460 & 521 & 1.67 & 0.87 & 0.80 & 60 & 137 \\
\hline
\end{tabular}

GGT: gamma-glutamil transferasa; FA: fosfatasa alcalina; ALT: alanino aminotransferasa; AST: aspartato aminotransferasa.

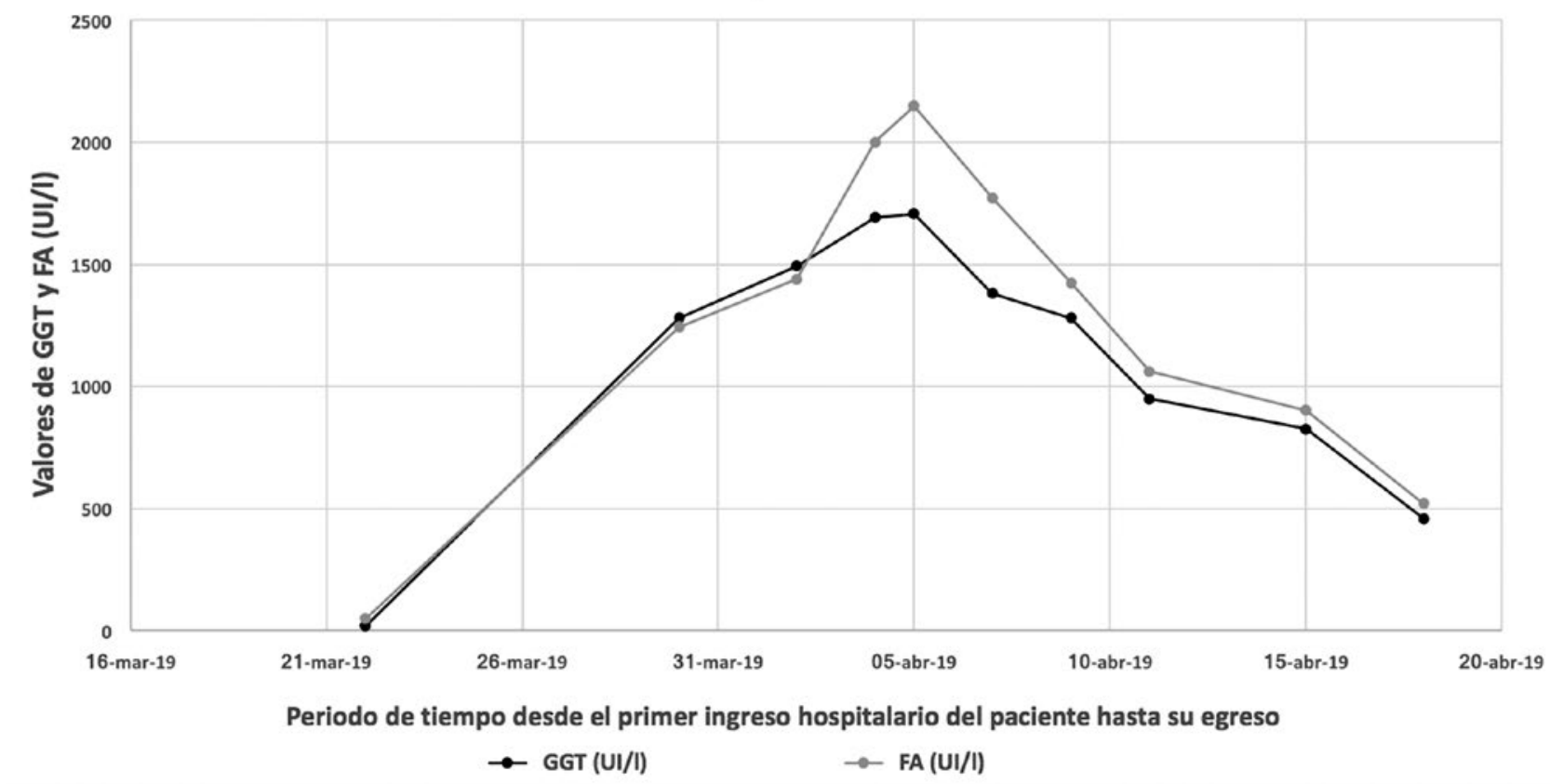

Figura 2. Representación de los valores de gamma-glutamil transferasa y fosfatasa alcalina desde el primer ingreso hospitalario del paciente (22 de marzo de 2019), suspensión de levodopa/carbidopa en su reingreso (5 de abril de 2019) hasta su egreso (18 de abril de 2019), que muestra un descenso gradual de ambas enzimas posterior a la retirada del fármaco. GGT: gamma-glutamil transferasa; FA: fosfatasa alcalina.

dicho fármaco el 5 de abril de 2019 y se inició pramipexol, además de metilprednisolona 60 mg cada 24 horas, con posterior reducción de dosis con prednisona secundaria a las manifestaciones de hipersensibilidad cutánea que presentó el paciente. Se llevó un control estricto de pruebas de función hepática, evidenciando un descenso gradual de estas (Tabla 1).

Su evolución fue favorable, con un descenso de la GGT y la FA mayor al 50\% en un periodo de 13 días posterior a la suspensión de I-dopa/carbidopa (Fig. 2).

\section{DISCUSIÓN Y CONCLUSIÓN}

La información que existe sobre la evolución de la presentación colestásica de DILI es limitada. La literatura actual muestra que del 5 al 13\% de los pacientes con DILI colestásica desarrollan enfermedad hepática crónica y que entre el 5 y el 14\% mueren o requieren trasplante por esta causa ${ }^{1-3}$.

A pesar de que la información epidemiológica sobre el efecto tóxico de los fármacos en el hígado es escasa, 
se sabe que la incidencia está aumentando de forma paralela a la introducción de nuevos medicamentos, al incremento de la esperanza de vida, la polifarmacia y al uso cada vez más extendido de productos herbolarios. La lesión hepática inducida por medicamentos puede ser intrínseca o idiosincrática, siendo más común esta última ${ }^{4,5}$. Es un problema clínico complejo de predecir, diagnosticar y tratar. Cuando se han descartado otras causas de hepatopatía y/o colestasis intrahepática, debe sospecharse e investigarse. Aunque no existe un examen o marcador que indique el diagnóstico absoluto de la enfermedad, se han desarrollado escalas y algoritmos que permiten valorar la probabilidad de DILI, y otras pruebas que por su complejidad y costo no son de uso habitual. Por lo anterior, es principalmente un diagnóstico de exclusión basado en evidencia circunstancial. El diagnóstico de certeza es complicado y la notificación por profesionales sanitarios de reacciones adversas a fármacos es escasa ${ }^{2,3}$.

La l-dopa es un precursor de aminoácidos de la dopamina y es el fármaco más eficaz y comúnmente utilizado en el tratamiento de la enfermedad de Parkinson. La I-dopa generalmente se combina con la carbidopa, que es un inhibidor de la I-aminoácido descarboxilasa, la enzima plasmática que metaboliza la I-dopa periféricamente. El tratamiento con la combinación de I-dopa y carbidopa se ha asociado con aumentos leves y transitorios de las enzimas hepáticas en una proporción de pacientes y con casos muy raros de lesión hepática aguda clínicamente evidente ${ }^{6}$.

Por lo general, la combinación se administra de tres a cuatro veces al día, aunque existe una forma de liberación controlada que permite dosificaciones diarias. La dosis óptima varía según el paciente. Por lo general, se inicia con una dosis baja y se incrementa según la respuesta clínica y la tolerancia. Los efectos secundarios pueden incluir náuseas, alucinaciones, confusión, hipotensión postural, sedación, estreñimiento, trastornos del sueño y depresión $n^{6,7}$.

«Se ha reportado que la combinación de I-dopa y carbidopa causa elevaciones de la aminotransferasa en suero hasta en el $9 \%$ de los pacientes, pero estas anomalías suelen ser leves, asintomáticas y autolimitadas. En raras ocasiones, las elevaciones de la aminotransferasa aumentan por encima de 5 a 10 veces el límite superior normal y requieren una interrupción o un ajuste de la dosis; sin embargo, son extremadamente raros los casos que cursan con elevación de bilirrubina total, fosfatasa alcalina y/o gamma glutamil transferasa» 7,8 .

En nuestro caso el paciente presentó una elevación importante de la FA y GGT, con elevación mínima de enzimas hepáticas, compatible con un patrón colestásico. Hubo una clara relación temporal entre el inicio/ fin del tratamiento y la aparición/desaparición de los síntomas, respectivamente. Además, se excluyeron otras causas de daño hepático. Con todo ello, y aplicando la escala CIOMS/RUCAM de valoración de hepatotoxicidad ${ }^{9,10}$, el caso obtuvo 9 puntos, lo que permite concluir como altamente probable o definitiva la hepatotoxicidad por consumo de dicho fármaco.

En la página de los Institutos Nacionales de Salud de EE.UU. (www.livertox.nih.gov), que nos describe casos de hepatotoxicidad de fármacos, así como el mecanismo del daño hepático que pueden ocasionar, no existen reportes publicados sobre el uso de I-dopa/carbidopa asociado a un patrón colestásico. La biopsia hepática está indicada ante un diagnóstico dudoso, ausencia de datos en la literatura sobre el fármaco sospechoso o como valoración pronóstica en las formas crónicas ${ }^{11}$. En nuestro paciente no se realizó dicho procedimiento, al definirse como un caso de DILI altamente probable o definitivo, presentando un descenso mayor del 50\% tanto de la FA como la GGT posterior a la suspensión de I-dopa/carbidopa en un periodo de 13 días.

La principal medida terapéutica es la inmediata suspensión de cualquier tratamiento farmacológico no esencial o sustitución del fármaco si fuera importante para su enfermedad, ya que la continuación del fármaco responsable de la hepatotoxicidad podría determinar una mayor probabilidad de evolución fulminante o crónica ${ }^{10}$. En el caso que presentamos el paciente tenía el diagnóstico de enfermedad de Parkinson, siendo el tratamiento con I-dopa/carbidopa importante para su padecimiento, por lo que fue sustituido por pramipexol. A pesar de que actualmente no existe algún medicamento que mejore completamente la evolución de la enfermedad, puede instaurarse un tratamiento dependiendo del tipo de daño que, asociado a la suspensión del fármaco causante, pudieran mejorar la evolución de la enfermedad y disminuir la mortalidad ${ }^{3,5}$.

La N-acetilcisteína ha mostrado una cierta eficacia en reducir la necesidad de trasplante en la insuficiencia hepática aguda no relacionada con intoxicación por 
paracetamol, de origen variado, a condición de que se administre en los grados iniciales de encefalopatía. Es posible que la $\mathrm{N}$-acetilcisteína pudiera también ser útil para prevenir la progresión de la hepatotoxicidad hacia formas fulminantes. Los corticosteroides podrían emplearse con fundamento en pacientes con manifestaciones de hipersensibilidad pronunciadas. Recientemente, un agonista del receptor farnesoide $\mathrm{X}$, el ácido obeticólico, que ha demostrado propiedades coleréticas y antifibróticas en modelos experimentales, ha sido ensayado con éxito para mejorar los índices de citolisis y colestasis en pacientes con cirrosis biliar primaria con respuesta deficiente al ácido ursodeoxicólico y podría ser, teóricamente, también útil en pacientes con colestasis tóxica prolongada ${ }^{12}$.

El uso de S-adenosilmetionina (SAMe) ha reportado una respuesta favorable en conjunto con el ácido ursodesoxicólico, donde se ha visto en cerca de 41 estudios que fueron incluidos en un metaanálisis para determinar la eficacia de la SAMe en la disminución del prurito y los valores séricos de bilirrubina, FA y GGT asociados a la colestasis, comparado con el placebo ${ }^{13}$.

El pronóstico a largo plazo para DILI, en general, depende de la presentación clínica y bioquímica inicial del paciente, siendo los niveles de aminotransferasas, de bilirrubina total, FA y GGT, junto con la prolongación de tiempos de coagulación y la presencia de encefalopatía hepática, los mayores predictores de mortalidad, la cual es del $10 \%$ en DILI colestásica ${ }^{13,14}$.

El interés de este caso radica en el abordaje para llegar al diagnóstico de DILI y en dar a conocer dicho efecto adverso de un patrón colestásico relacionado al uso de I-dopa/carbidopa, existiendo escasos datos en la literatura en relación con la hepatoxicidad de este fármaco. Este caso fue evaluado de manera multidisciplinaria, es decir, por personal médico del servicio de medicina interna, médico geriatra y un farmacéutico de hospital, con la finalidad de dar también cumplimiento a la norma Oficial Mexicana NOM-220-SSA1-2016, Instalación y Operación de la Farmacovigilancia.

Se realizó la evaluación de la causalidad, la cual, de acuerdo con la Organización Mundial de la Salud, permite estimar la probabilidad de atribuir a un medicamento la reacción adversa observada con base en categorías probabilísticas. En este caso se clasificó como «posible», ya que existió una alteración de la función hepática que pudo comprobarse en los valores de laboratorio fuera de rangos normales. Además, se observó una secuencia temporal con la administración del medicamento sospechoso, la dosis y la aparición de la sospecha de reacción adversa, al igual que a la suspensión del medicamento sospechoso, continuando con la administración de los otros medicamentos concomitantes y presentando una mejoría de la sintomatología. No se tuvo una readministración del medicamento I-dopa/carbidopa, ni datos sobre una reacción similar que hubiera sufrido el paciente con la exposición previa al medicamento.

Este caso fue clasificado como "grave" y de acuerdo con la severidad, que se relaciona con la intensidad de la manifestación clínica, se consideró como «severo», ya que requirió tratamiento farmacológico y suspensión del medicamento causante. El análisis del caso con un enfoque multidisciplinario permitió fomentar la notificación de los problemas de seguridad con el uso de medicamentos entre todos los profesionales de salud y el reporte de casos al Programa Permanente de Farmacovigilancia en México. Se mantuvo la confidencialidad de la información del paciente y se notificó el caso con un grado de calidad elevado (grado 2), lo que implica que se tengan datos suficientes sobre el medicamento sospechoso y del evento, así como el uso de la terminología MedDRA para la descripción de las manifestaciones clínicas, garantizando que esta información podrá formar parte de las estadísticas nacionales e internacionales y de la literatura médica científica.

\section{AGRADECIMIENTOS}

A las divisiones de Medicina Interna, Geriatría, Hepatología y de Farmacovigilancia del Hospital General Dr. Manuel Gea González, Ciudad de México, México.

\section{FINANCIAMIENTO}

La presente investigación no ha recibido ninguna beca específica de agencias de los sectores público, comercial o sin ánimo de lucro.

\section{CONFLICTO DE INTERESES}

No existen conflictos de intereses por parte del autor y coautores de este artículo. 


\section{RESPONSABILIDADES ÉTICAS}

Protección de personas y animales. Los autores declaran que para esta investigación no se han realizado experimentos en seres humanos ni en animales.

Confidencialidad de los datos. Los autores declaran que han seguido los protocolos de su centro de trabajo sobre la publicación de datos de pacientes.

\section{Derecho a la privacidad y consentimiento informado.} Los autores han obtenido el consentimiento informado de los pacientes y/o sujetos referidos en el artículo. Este documento obra en poder del autor de correspondencia.

\section{BIBLIOGRAFÍA}

1. Chalasani NP, Hayashi PH, Bonkovsky HL, Navarro VJ, Lee WM, Fontana RJ. ACG Clinical Guideline: the diagnosis and management of idiosyncratic drug-induced liver injury. Am J Gastroenterol. 2014;109:950-66.

2. Khashab M, Tector AJ, Kwo PY. Epidemiology of acute liver failure. Curr Gastroenterol Rep. 2007;9:66-73.
3. Díaz-García JD, Córdova-Gallardo J, Torres-Viloria A, Estrada-Hernández A, Torre-Delgadillo A. Lesión hepática inducida por fármacos secundaria al uso de esteroides anabólicos. Rev Gastroenterol Mex. 2019;85(1):92-4.

4. Danan G. Benichou C. Causality assessment of adverse reactions to drugs-I. A novel method based on the conclusions of international consensus meetings: Application to drug-induced liver injuries. J Clin Epidemiol. 2014;46:1323-30.

5. Kaplowitz N. Idiosyncratic drug hepatotoxicity. Nat Rev Drug Discov. 2005;4:489-98.

6. Brooks DJ, Leinonen M, Kuoppamäki M, Nissinen H. Five-year efficacy and safety of levodopa/DDCl and entacapone in patients with Parkinson's disease. J Neural Transm. 2008:115:843-9.

7. Hernández N, Bessone F, Sánchez A, di Pace M, Brahm J, Zapata R, et al. Profile of idiosyncratic drug induced liver injury in Latin America: An analysis of published reports. Ann Hepatol. 2014;13:231-9.

8. Chalasani N, Bonkovsky HL, Fontana R, Lee W, Stolz A, Talwalkar J, et al. United States Drug Induced Liver Injury Network. Features and outcomes of 899 patients with drug-induced liver injury: The DILIN Prospective Study. Gastroenterology. 2015;148:1340-52.

9. Maddur H, Chalasani N. Idiosyncratic drug-induced liver injury: A clinical update. Curr Gastroenterol Rep. 2011;13:65-71.

10. Bjornsson ES, Jonasson JG. Drug-induced cholestasis. Clin Liver Dis. 2013:17(2):191-209.

11. Ishak K, Baptista A, Bianchi L. Histological grading and staging of chronic hepatitis. J Hepatol. 1995;22:696-9.

12. Chughlay MF, Kramer N, Werfalli M, Spearman W, Engel ME, Cohen K. Nacetylcysteine for non-paracetamol drug-induced liver injury: a systematic review protocol. Syst Rev. 2015;4(84):1-6.

13. Clinical Guidelines and Recommendations. S-Adenosyl-L-methionine for treatments of depression, osteoarthritis, and liver disease [Internet]. Agency for Healthcare Research and Quality [fecha de publicación: junio de 2011]. Disponible en: https://effectivehealthcare.ahrq.gov/sites/default/files/ $\mathrm{pdf} /$ trial-quality-effect-evidence research.pdf

14. Rambaldi A, Gluud C. S-adenosyl-L-methionine for alcoholic liver disease. Cochrane Database Syst Rev. 2001;4:CD002235 